Network Working Group

Request for Comments: 2963

Category: Informational
O. Bonaventure

FUNDP

S. De Cnodder

Alcatel

October 2000

\title{
A Rate Adaptive Shaper for Differentiated Services
}

Status of this Memo

This memo provides information for the Internet community. It does not specify an Internet standard of any kind. Distribution of this memo is unlimited.

Copyright Notice

Copyright (C) The Internet Society (2000). All Rights Reserved.

Abstract

This memo describes several Rate Adaptive Shapers (RAS) that can be used in combination with the single rate Three Color Markers (srTCM) and the two rate Three Color Marker (trTCM) described in RFC2697 and RFC2698, respectively. These RAS improve the performance of TCP when a TCM is used at the ingress of a diffserv network by reducing the burstiness of the traffic. With TCP traffic, this reduction of the burstiness is accompanied by a reduction of the number of marked packets and by an improved TCP goodput. The proposed RAS can be used at the ingress of Diffserv networks providing the Assured Forwarding Per Hop Behavior (AF PHB). They are especially useful when a TCM is used to mark traffic composed of a small number of TCP connections.

1. Introduction

In DiffServ networks [RFC2475], the incoming data traffic, with the $A F$ PHB in particular, could be subject to marking where the purpose of this marking is to provide a low drop probability to a minimum part of the traffic whereas the excess will have a larger drop probability. Such markers are mainly token bucket based such as the single rate Three Color Marker (srTCM) and two rate Three Color Marker (trTCM) described in [RFC2697] and [RFC2698], respectively.

Similar markers were proposed for ATM networks and simulations have shown that their performance with TCP traffic was not always satisfactory and several researchers have shown that these performance problems could be solved in two ways: 
1. increasing the burst size, i.e. increasing the Committed Burst Size (CBS) and the Peak Burst Size (PBS) in case of the trTCM, or

2. shaping the traffic such that a part of the burstiness is removed.

The first solution has as major disadvantage that the traffic sent to the network can be very bursty and thus engineering the network to provide a low packet loss ratio can become difficult. To efficiently support bursty traffic, additional resources such as buffer space are needed. Conversely, the major disadvantage of shaping is that the traffic encounters additional delay in the shaper's buffer.

In this document, we propose two shapers that can reduce the burstiness of the traffic upstream of a TCM. By reducing the burstiness of the traffic, the adaptive shapers increase the percentage of packets marked as green by the TCM and thus the overall goodput of the users attached to such a shaper.

Such rate adaptive shapers will probably be useful at the edge of the network (i.e. inside access routers or even network adapters). The simulation results in [Cnodder] show that these shapers are particularly useful when a small number of TCP connections are processed by a TCM.

The structure of this document follows the structure proposed in [Nichols]. We first describe two types of rate adaptive shapers in section two. These shapers correspond to respectively the srTCM and the trTCM. In section 3, we describe an extension to the simple shapers that can provide a better performance. We briefly discuss simulation results in the appendix.

2. Description of the rate adaptive shapers

\subsection{Rate adaptive shaper}

The rate adaptive shaper is based on a similar shaper proposed in [Bonaventure] to improve the performance of TCP with the Guaranteed Frame Rate [TM41] service category in ATM networks. Another type of rate adaptive shaper suitable for differentiated services was briefly discussed in [Azeem]. A RAS will typically be used as shown in figure 1 where the meter and the marker are the TCMs proposed in [RFC2697] and [RFC2698]. 


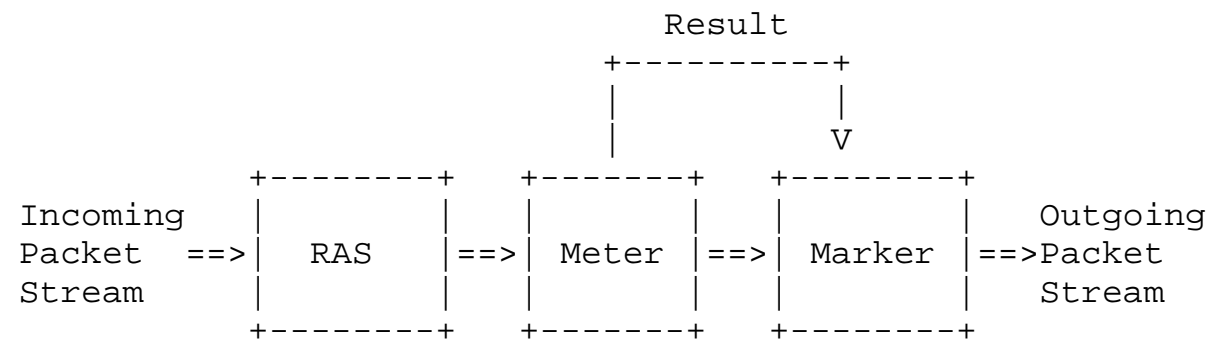

Figure 1. Rate adaptive shaper

The presentation of the rate adaptive shapers in Figure 1 is somewhat different as described in [RFC2475] where the shaper is placed after the meter. The main objective of the shaper is to produce at its output a traffic that is less bursty than the input traffic, but the shaper avoids to discard packets in contrast with classical token bucket based shapers. The shaper itself consists of a tail-drop FIFO queue which is emptied at a variable rate. The shaping rate, i.e. the rate at which the queue is emptied, is a function of the occupancy of the FIFO queue. If the queue occupancy increases, the shaping rate will also increase in order to prevent loss and too large delays through the shaper. The shaping rate is also a function of the average rate of the incoming traffic. The shaper was designed to be used in conjunction with meters such as the TCMs proposed in [RFC2697] and [RFC2698].

There are two types of rate adaptive shapers. The single rate rate adaptive shaper (srRAS) will typically be used upstream of a srTCM while the two rates rate adaptive shaper (trRAS) will usually be used upstream of a trTCM.

\subsection{Configuration of the srRAS}

The srRAS is configured by specifying four parameters: the Committed Information Rate (CIR), the Maximum Information Rate (MIR) and two buffer thresholds: CIR_th (Committed Information Rate threshold) and MIR_th (Maximum Information Rate threshold). The CIR shall be specified in bytes per second and MUST be configurable. The MIR shall be specified in the same unit as the CIR and SHOULD be configurable. To achieve a good performance, the CIR of a srRAS will usually be set to the same value as the CIR of the downstream srTCM. A typical value for the MIR would be the line rate of the output link of the shaper. When the CIR and optionally the MIR are configured, the srRAS MUST ensure that the following relation is verified: 


$$
\text { CIR }<=\text { MIR }<=\text { line rate }
$$

The two buffer thresholds, CIR_th and MIR_th shall be specified in bytes and SHOULD be configurable. If these thresholds are configured, then the srRAS MUST ensure that the following relation holds:

$$
\text { CIR_th }<=\text { MIR_th }<=\text { buffer size of the shaper }
$$

The chosen values for CIR_th and MIR_th will usually depend on the values chosen for CBS and PBS in the downstream srTCM. However, this dependency does not need to be standardized.

\subsection{Behavior of the srRAS}

The output rate of the shaper is based on two factors. The first one is the (long term) average rate of the incoming traffic. This average rate can be computed by several means. For example, the function proposed in [Stoica] can be used (i.e. EARnew = [ (1-exp ($\left.\mathrm{T} / \mathrm{K}))^{\star} \mathrm{L} / \mathrm{T}\right]+\exp (-\mathrm{T} / \mathrm{K}){ }^{*}$ EARold where EARold is the previous value of the Estimated Average Rate, EARnew is the updated value, $\mathrm{K}$ a constant, L the size of the arriving packet and $\mathrm{T}$ the amount of time since the arrival of the previous packet). Other averaging functions can be used as well.

The second factor is the instantaneous occupancy of the FIFO buffer of the shaper. When the buffer occupancy is below CIR_th, the output rate of the shaper is set to the maximum of the estimated average rate (EAR(t)) and the CIR. This ensures that the shaper buffer will be emptied at least at a rate equal to CIR. When the buffer occupancy increases above CIR_th, the output rate of the shaper is computed as the maximum of the EAR(t) and a linear function $F$ of the buffer occupancy for which F (CIR_th)=CIR and F (MIR_th)=MIR. When the buffer occupancy reaches the MIR_th threshold, the output rate of the shaper is set to the maximum information rate. The computation of the shaping rate is illustrated in figure 2. We expect that real implementations will only use an approximate function to compute the shaping rate. 


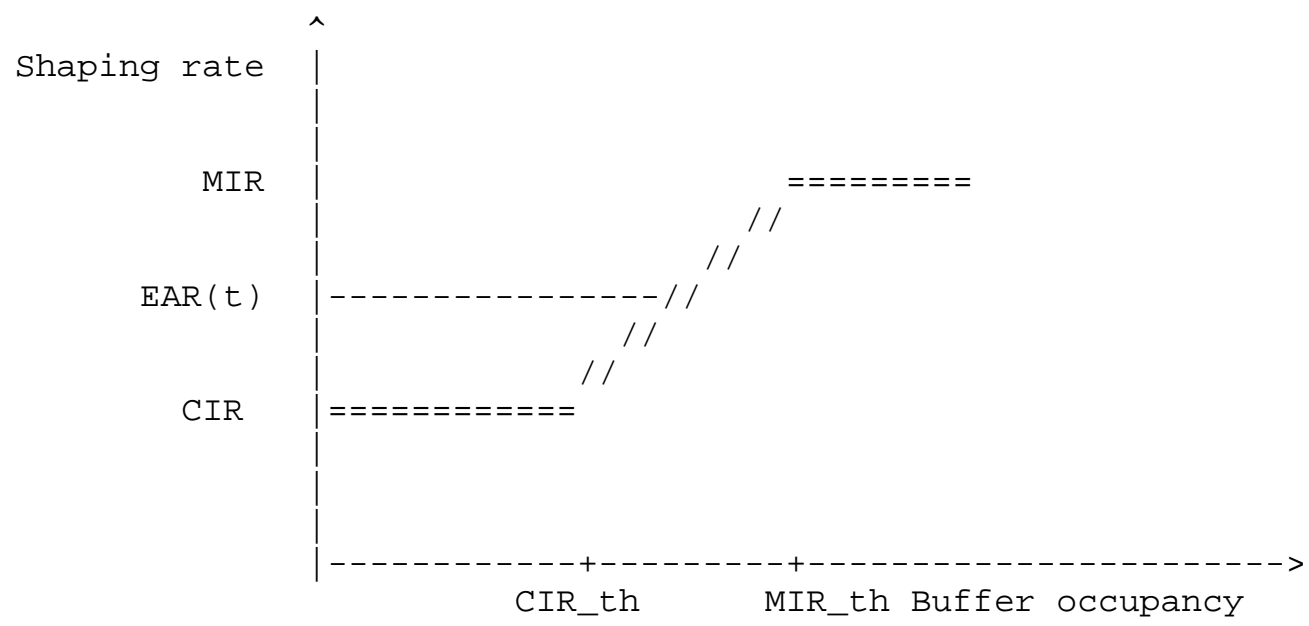

Figure 2. Computation of shaping rate for srRAS

\subsection{Configuration of the trRAS}

The trRAS is configured by specifying six parameters: the Committed Information Rate (CIR), the Peak Information Rate (PIR), the Maximum Information Rate (MIR) and three buffer thresholds: CIR_th, PIR_th and MIR_th. The CIR shall be specified in bytes per second and MUST be configurable. To achieve a good performance, the CIR of a trRAS will usually be set at the same value as the CIR of the downstream trTCM. The PIR shall be specified in the same unit as the CIR and MUST be configurable. To achieve a good performance, the PIR of a trRAS will usually be set at the same value as the PIR of the downstream trRAS. The MIR SHOULD be configurable and shall be specified in the same unit as the CIR. A typical value for the MIR will be the line rate of the output link of the shaper. When the values for CIR, PIR and optionally MIR are configured, the trRAS MUST ensure that the following relation is verified:

$$
\text { CIR }<=\text { PIR }<=\text { MIR }<=\text { line rate }
$$

The three buffer thresholds, CIR_th, PIR_th and MIR_th shall be specified in bytes and SHOULD be configurable. If these thresholds are configured, then the trRAS MUST ensure that the following relation is verified:

CIR_th $<=$ PIR_th $<=$ MIR_th $<=$ buffer size of the shaper

The CIR_th, PIR_th and MIR_th will usually depend on the values chosen for the CBS and the PBS in the downstream trTCM. However, this dependency does not need to be standardized. 


\subsection{Behavior of the trRAS}

The output rate of the trRAS is based on two factors. The first is the (long term) average rate of the incoming traffic. This average rate can be computed as for the srRAS.

The second factor is the instantaneous occupancy of the FIFO buffer of the shaper. When the buffer occupancy is below CIR_th, the output rate of the shaper is set to the maximum of the estimated average rate (EAR(t)) and the CIR. This ensures that the shaper will always send traffic at least at the CIR. When the buffer occupancy increases above CIR_th, the output rate of the shaper is computed as the maximum of the EAR(t) and a piecewise linear function $F$ of the buffer occupancy. This piecewise function can be defined as follows. The first piece is between zero and CIR_th where $F$ is equal to CIR. This means that when the buffer occupancy is below a certain threshold CIR_th, the shaping rate is at least CIR. The second piece is between CIR_th and PIR_th where $F$ increases linearly from CIR to PIR. The third part is from PIR_th to MIR_th where F increases linearly from PIR to the MIR and finally when the buffer occupancy is above MIR_th, the shaping rate remains constant at the MIR. The computation of the shaping rate is illustrated in figure 3 . We expect that real implementations will use an approximation of the function shown in this figure to compute the shaping rate.

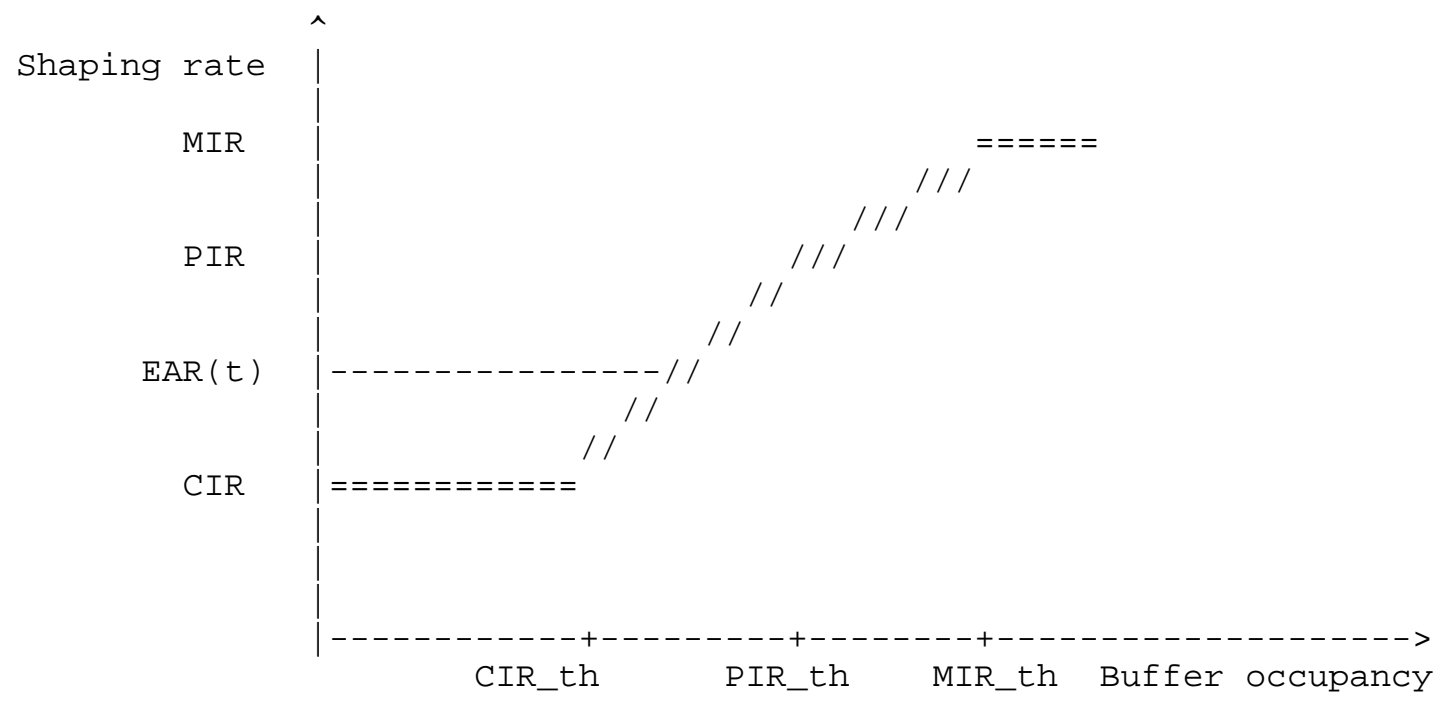

Figure 3. Computation of shaping rate for trRAS 
3. Description of the green RAS.

3.1. The green rate adaptive shapers

The srRAS and the trRAS described in the previous section are not aware of the status of the meter. This entails that a RAS could unnecessarily delay a packet although there are sufficient tokens available to color the packet green. This delay could mean that TCP takes more time to increase its congestion window and this may lower the performance with TCP traffic. The green RAS shown in figure 4 solves this problem by coupling the shaper with the meter.

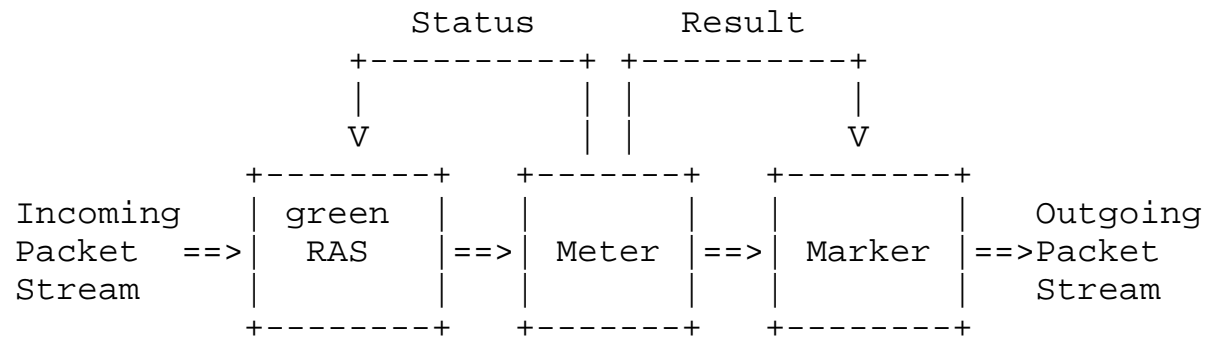

Figure 4. green RAS

The two rate adaptive shapers described in section 2 calculate a shaping rate, which is defined as the maximum of the estimated average incoming data rate and some function of the buffer occupancy. Using this shaping rate, the RAS computes the time schedule at which the packet at the head of the queue of the shaper is to be released. The main idea of the green RAS is to couple the shaper with the downstream meter so that the green RAS knows at what time the packet at the head of its queue would be accepted as green by the meter. If this time instant is earlier than the release time computed from the current shaping rate, then the packet can be released at this time instant. Otherwise, the packet at the head of the queue of the green RAS will be released at the time instant calculated from the current shaping rate.

3.2. Configuration of the Green single rate Rate Adaptive Shaper (GsrRAS)

The G-srRAS must be configured in the same way as the srRAS (see section 2.2). 


\subsection{Behavior of the G-srRAS}

First of all, the shaping rate of the G-srRAS is calculated in the same way as for the srRAS. With the srRAS, this shaping rate determines a time schedule, T1, at which the packet at the head of the queue is to be released from the shaper.

A second time schedule, T2, is calculated as the earliest time instant at which the packet at the head of the shaper's queue would be colored as green by the downstream srTCM. Suppose that a packet of size B bytes is at the head of the shaper and that CIR is the Committed Information Rate of the srTCM in bytes per second. If we denote the current time by $t$ and by Tc(t) the amount of green tokens in the token bucket of the srTCM at time $t$, then $T 2$ is equal to $\max (t, t+(B-T C(t)) / C I R)$. If $B$ is larger than CBS, the committed Burst Size of the srTCM, then $\mathrm{T} 2$ is set to infinity.

When a packet arrives at the head of the queue of the shaper, it will leave this queue not sooner than min(T1, T2) from the shaper.

\subsection{Configuration of the Green two rates Rate Adaptive Shaper (G-trRAS)}

The G-trRAS must be configured in the same way as the trRAS (see section 2.4).

\subsection{Behavior of the G-trRAS}

First of all, the shaping rate of the G-trRAS is calculated in the same way as for the trRAS. With the trRAS, this shaping rate determines a time schedule, T1, at which the packet at the head of the queue is to be released from the shaper.

A second time schedule, T2, is calculated as the earliest time instant at which the packet at the head of the shaper's queue would be colored as green by the downstream trTCM. Suppose that a packet of size B bytes is at the head of the shaper and that CIR is the Committed Information Rate of the srTCM in bytes per second. If we denote the current time by $t$ and by $T c(t)$ (resp. Tp(t)) the amount of green (resp. yellow) tokens in the token bucket of the trTCM at time $t$, then T2 is equal to $\max (t, t+(B-T C(t)) / C I R, t+(B-T p(t)) / P I R)$. If $B$ is larger than CBS, the committed burst size, or PBS, the peak burst size, of the srTCM, then $\mathrm{T} 2$ is set to infinity.

When a packet arrives at the head of the queue of the shaper, it will leave this queue not sooner than min(T1, T2) from the shaper. 
4. Assumption

The shapers discussed in this document assume that the Internet traffic is dominated by protocols such as TCP that react appropriately to congestion by decreasing their transmission rate.

The proposed shapers do not provide a performance gain if the traffic is composed of protocols that do not react to congestion by decreasing their transmission rate.

5. Example services

The shapers discussed in this document can be used where the TCMs proposed in [RFC2697] and [RFC2698] are used. In fact, simulations briefly discussed in Appendix A show that the performance of TCP can be improved when a rate adaptive shaper is used upstream of a TCM. We expect such rate adaptive shapers to be particularly useful at the edge of the network, for example inside (small) access routers or even network adapters.

6. The rate adaptive shaper combined with other markers

This document explains how the idea of a rate adaptive shaper can be combined with the srTCM and the trTCM. This resulted in the srRAS and the G-srRAS for the srTCM and in the trRAS and the G-trRAS for the trTCM. Similar adaptive shapers could be developed to support other traffic markers such as the Time Sliding Window Three Color Marker (TSWTCM) [Fang]. However, the exact definition of such new adaptive shapers and their performance is outside the scope of this document.

7. Security Considerations

The shapers described in this document have no known security concerns.

8. Intellectual Property Rights

The IETF has been notified of intellectual property rights claimed in regard to some or all of the specification contained in this document. For more information consult the online list of claimed rights.

9. Acknowledgement

We would like to thank Emmanuel Desmet for his comments. 
10. References

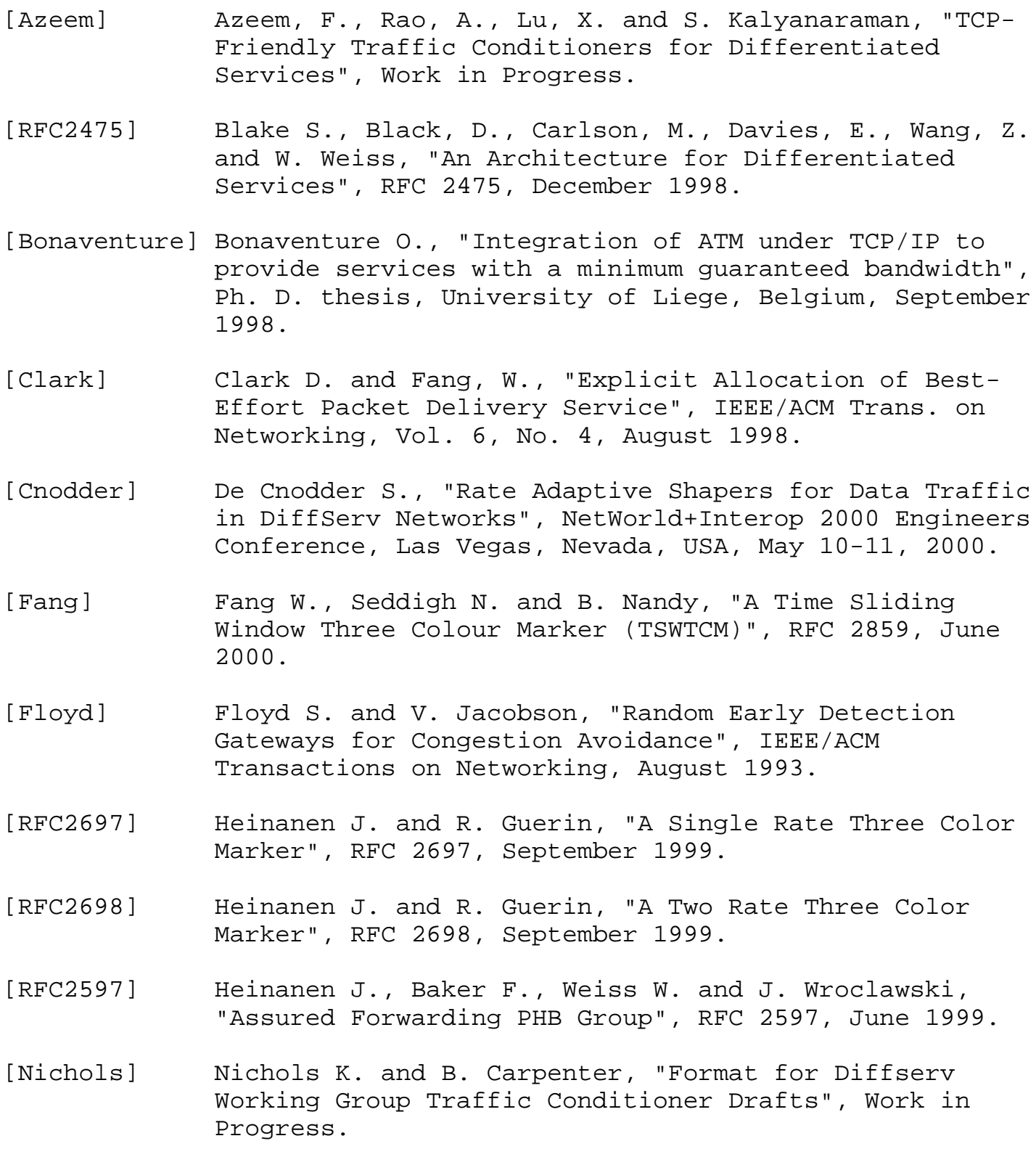


[Stoica] Stoica I., Shenker S. and H. Zhang, "Core-stateless fair queueuing: achieving approximately fair bandwidth allocations in high speed networks", ACM SIGCOMM98, pp. 118-130, sept. 1998

[TM41] ATM Forum, Traffic Management Specification, verion $4.1,1999$ 


\section{Appendix}

A. Simulation results

We briefly discuss simulations showing the benefits of the proposed shapers in simple network environments. Additional simulation results may be found in [Cnodder].

A.1 description of the model

To evaluate the rate adaptive shaper through simulations, we use the simple network model depicted in Figure A.1. In this network, we consider that a backbone network is used to provide a LAN

Interconnection service to ten pairs of LANs. Each LAN corresponds to an uncongested switched $10 \mathrm{Mbps}$ LAN with ten workstations attached to a customer router ( $1-\mathrm{C} 10$ in figure $\mathrm{A} .1)$. The delay on the LAN links is set to $1 \mathrm{msec}$. The MSS size of the workstations is set to 1460 bytes. The workstations on the left hand side of the figure send traffic to companion workstations located on the right hand side of the figure. All traffic from the LAN attached to customer router $\mathrm{C} 1$ is sent to the LAN attached to customer router $\mathrm{Cl}^{\prime}$. There are ten workstations on each LAN and each workstation implements SACK-TCP with a maximum window size of 64 kBytes. 


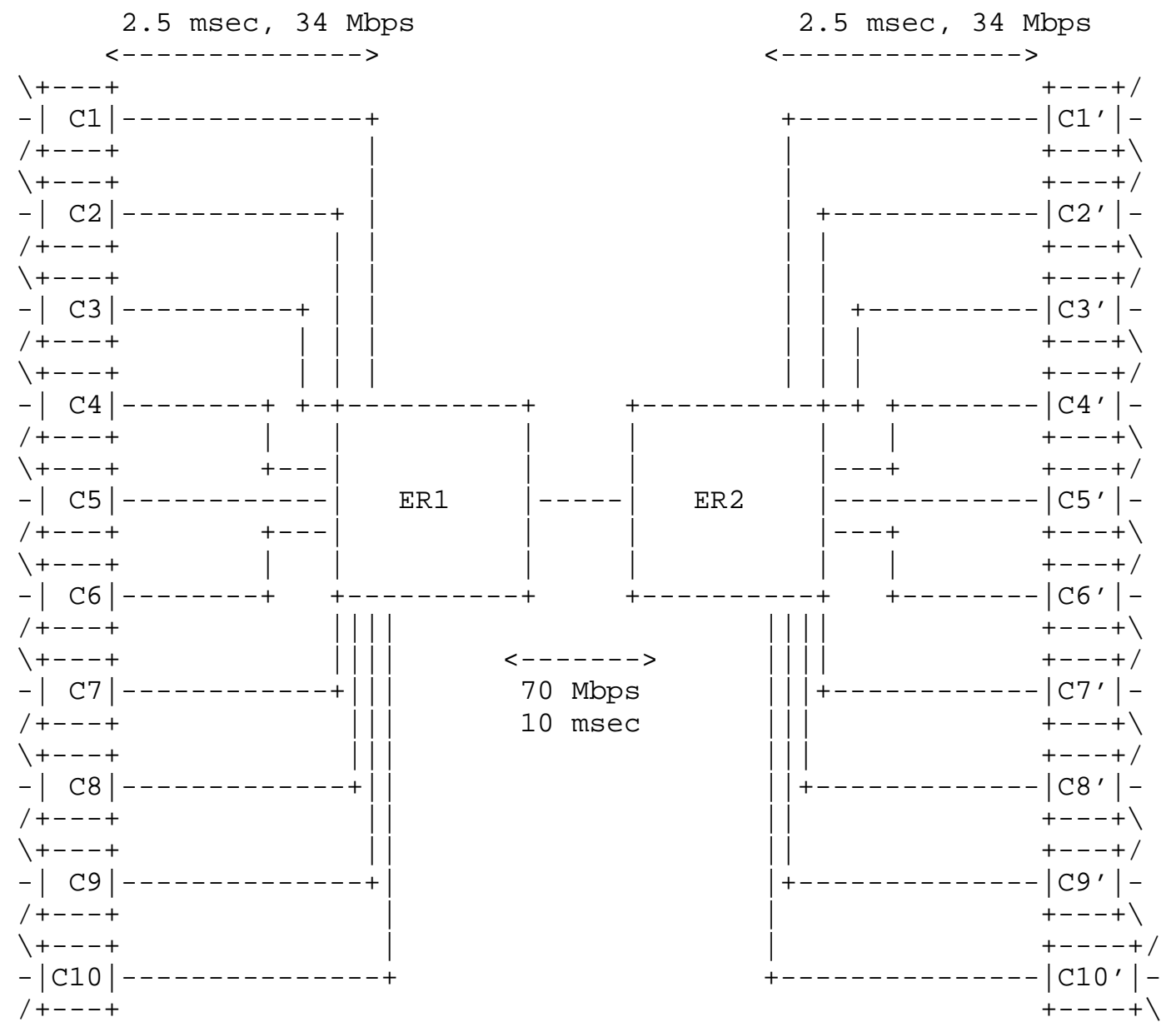

Figure A.1. the simulation model.

The customer routers are connected with $34 \mathrm{Mbps}$ links to the backbone network which is, in our case, composed of a single bottleneck 70 Mbps link between the edge routers ER1 and ER2. The delay on all the customer-edge $34 \mathrm{Mbps}$ links has been set to 2.5 msec to model a MAN or small WAN environment. These links and the customer routers are not a bottleneck in our environment and no losses occurs inside the edge routers. The customer routers are equipped with a trTCM [Heinanen2] and mark the incoming traffic. The parameters of the trTCM are shown in table A.1. 
Table A.1: configurations of the trTCMs

$\begin{array}{lrrr}\text { Router } & \text { CIR } & \text { PIR } & \text { Line Rate } \\ \text { C1 } & 2 \text { Mbps } & 4 \mathrm{Mbps} & 34 \mathrm{Mbps} \\ \text { C2 } & 4 \mathrm{Mbps} & 8 \mathrm{Mbps} & 34 \mathrm{Mbps} \\ \text { C3 } & 6 \mathrm{Mbps} & 12 \mathrm{Mbps} & 34 \mathrm{Mbps} \\ \text { C4 } & 8 \mathrm{Mbps} & 16 \mathrm{Mbps} & 34 \mathrm{Mbps} \\ \text { C5 } & 10 \mathrm{Mbps} & 20 \mathrm{Mbps} & 34 \mathrm{Mbps} \\ \text { C6 } & 2 \mathrm{Mbps} & 4 \mathrm{Mbps} & 34 \mathrm{Mbps} \\ \text { C7 } & 4 \mathrm{Mbps} & 8 \mathrm{Mbps} & 34 \mathrm{Mbps} \\ \text { C8 } & 6 \mathrm{Mbps} & 12 \mathrm{Mbps} & 34 \mathrm{Mbps} \\ \text { C9 } & 8 \mathrm{Mbps} & 16 \mathrm{Mbps} & 34 \mathrm{Mbps} \\ \text { C10 } & 10 \mathrm{Mbps} & 20 \mathrm{Mbps} & 34 \mathrm{Mbps}\end{array}$

All customer routers are equipped with a trTCM where the CIR are 2 Mbps for router $\mathrm{C} 1$ and $\mathrm{C} 6,4 \mathrm{Mbps}$ for $\mathrm{C} 2$ and C7, $6 \mathrm{Mbps}$ for $\mathrm{C} 3$ and $\mathrm{C} 8,8 \mathrm{Mbps}$ for $\mathrm{C} 4$ and $\mathrm{C} 9$ and $10 \mathrm{Mbps}$ for $\mathrm{C} 5$ and $\mathrm{C} 10$. Routers C6-C10 also contain a trRAS in addition to the trTCM while routers $\mathrm{C} 1-\mathrm{C} 5$ only contain a trTCM. In all simulations, the PIR is always twice as large as the CIR. Also the PBS is the double of the CBS. The CBS will be varied in the different simulation runs.

The edge routers, ER1 and ER2, are connected with a $70 \mathrm{Mbps}$ link which is the bottleneck link in our environment. These two routers implement the RIO algorithm [Clark] that we have extended to support three drop priorities instead of two. The thresholds of the parameters are 100 and 200 packets (minimum and maximum threshold, respectively) for the red packets, 200 and 400 packets for the yellow packets and 400 and 800 for the green packets. These thresholds are reasonable since there are 100 TCP connections crossing each edge router. The parameter maxp of RIO for green, yellow and red are respectively set to $0.02,0.05$, and 0.1 . The weight to calculate the average queue length which is used by RED or RIO is set to 0.002 [Floyd].

The simulated time is set to 102 seconds where the first two seconds are not used to gather TCP statistics (the so-called warm-up time) such as goodput.

\section{A.2 Simulation results for the trRAS}

For our first simulations, we consider that routers C1-C5 only utilize a trTCM while routers C6-C10 utilize a rate adaptive shaper in conjunction with a trTCM. All routers use a CBS of 3 kBytes. In table A.2, we show the total throughput achieved by the workstations attached to each LAN as well as the total throughput for the green and the yellow packets as a function of the CIR of the trTCM used on the customer router attached to this LAN. The throughput of the red 
packets is equal to the difference between the total traffic and the green and the yellow traffic. In table A.3, we show the total throughput achieved by the workstations attached to customer routers with a rate adaptive shaper.

Table A.2: throughput in Mbps for the unshaped traffic.

\begin{tabular}{|c|c|c|c|c|}
\hline & & green & yellow & total \\
\hline 2Mbps & {$[\mathrm{C} 1]$} & 1.10 & 0.93 & 2.25 \\
\hline $4 \mathrm{Mbps}$ & [C2 ] & 2.57 & 1.80 & 4.55 \\
\hline $6 \mathrm{Mbps}$ & [C3 ] & 4.10 & 2.12 & 6.39 \\
\hline 8Mbps & [C4 ] & 5.88 & 2.32 & 8.33 \\
\hline $10 \mathrm{Mbps}$ & [C5 ] & 7.57 & 2.37 & 10.0 \\
\hline
\end{tabular}

Table A.3: throughput in Mbps for the adaptively shaped traffic.

$\begin{array}{lllll}\text { 2Mbps [C6] } & 2.00 & \text { green } & \text { yellow } & \text { total } \\ 4 \mathrm{Mbps} \text { [C7] } & 3.97 & 1.69 & 3.71 \\ \text { 6Mbps [C8] } & 5.93 & 2.34 & 6.33 \\ \text { 8Mbps [C9] } & 7.84 & 2.23 & 8.17 \\ \text { 10Mbps [C10] } 9.77 & 2.28 & 10.1\end{array}$

This first simulation shows clearly that the workstations attached to an edge router with a rate adaptive shaper have a clear advantage, from a performance point of view, with respect to workstations attached to an edge router with only a trTCM. The performance improvement is the result of the higher proportion of packets marked as green by the edge routers when the rate adaptive shaper is used.

To evaluate the impact of the CBS on the TCP goodput, we did additional simulations were we varied the CBS of all customer routers.

Table A.4 shows the total goodput for workstations attached to, respectively, routers C1 (trTCM with 2 Mbps CIR, no adaptive shaping), C6 (trRAS with 2 Mbps CIR and adaptive shaping), C3 (trTCM with 6 Mbps CIR, no adaptive shaping), and C8 (trRAS with 6 Mbps CIR and adaptive shapingl for various values of the CBS. From this table, it is clear that the rate adaptive shapers provide a performance benefit when the CBS is small. With a very large CBS, the performance decreases when the shaper is in use. However, a CBS of a few hundred KBytes is probably too large in many environments. 


\begin{tabular}{|c|c|c|c|c|}
\hline CBS & 2_Mbps_unsh & 2_Mbps_sh & 6_Mbps_unsh & 6_Mbps_sh \\
\hline 3 & 1.88 & $3.4 \overline{9}$ & 5.91 & 7.77 \\
\hline 10 & 2.97 & 2.91 & 6.76 & 7.08 \\
\hline 25 & 3.14 & 2.78 & 7.07 & 6.73 \\
\hline 50 & 3.12 & 2.67 & 7.20 & 6.64 \\
\hline 75 & 3.18 & 2.56 & 7.08 & 6.58 \\
\hline 100 & 3.20 & 2.64 & 7.00 & 6.62 \\
\hline 150 & 3.21 & 2.54 & 7.11 & 6.52 \\
\hline 200 & 3.26 & 2.57 & 7.07 & 6.53 \\
\hline 300 & 3.19 & 2.53 & 7.13 & 6.49 \\
\hline 400 & 3.13 & 2.48 & 7.18 & 6.43 \\
\hline
\end{tabular}

A.3 Simulation results for the Green trRAS

We use the same scenario as in A.2 but now we use the Green trRAS (G-trRAS) .

Table A.5 and Table A.6 show the results of the same scenario as for Table A.2 and Table A.3 but the shaper is now the G-trRAS. We see that the shaped traffic performs again much better, also compared to the previous case (i.e. where the trRAS was used). This is because the amount of yellow traffic increases with the expense of a slight decrease in the amount of green traffic. This can be explained by the fact that the G-trRAS introduces some burstiness.

\begin{tabular}{|c|c|c|c|c|}
\hline & & green & yellow & total \\
\hline 2Mbps & [C1 ] & 1.10 & 0.95 & 2.26 \\
\hline $4 \mathrm{Mbps}$ & [C2 ] & 2.41 & 1.66 & 4.24 \\
\hline $6 \mathrm{Mbps}$ & [C3 ] & 3.94 & 1.97 & 6.07 \\
\hline $8 \mathrm{Mbps}$ & [C4 ] & 5.72 & 2.13 & 7.96 \\
\hline $10 \mathrm{Mbps}$ & [C5 ] & 7.25 & 2.29 & 9.64 \\
\hline
\end{tabular}

Table A.6: throughput in Mbps for the adaptively shaped traffic.

\begin{tabular}{|c|c|c|c|c|}
\hline & & green & yellow & total \\
\hline 2Mbps & [C6] & 1.92 & 1.75 & 3.77 \\
\hline 4Mbps & [C7 ] & 3.79 & 3.24 & 7.05 \\
\hline $6 \mathrm{Mbps}$ & [C8 ] & 5.35 & 3.62 & 8.97 \\
\hline $8 \mathrm{Mbps}$ & [C9] & 6.96 & 3.48 & 10.4 \\
\hline $10 \mathrm{Mbps}$ & [C10] & 8.69 & 3.06 & 11.7 \\
\hline
\end{tabular}

The impact of the CBS is shown in Table A.7 which is the same scenario as Table A.4 with the only difference that the shaper is now the G-trRAS. We see that the shaped traffic performs much better than the unshaped traffic when the CBS is small. When the CBS is 
large, the shaped and unshaped traffic performs more or less the same. This is in contrast with the trRAS, where the performance of the shaped traffic was slightly worse in case of a large CBS.

Table A.7: goodput in Mbps (link rate is 70 Mbps) versus CBS in KBytes.

$\begin{array}{lcccc}\text { CBS } & \text { 2_Mbps_unsh } & \text { 2_Mbps_sh } & \text { 6_Mbps_unsh } & \text { 6_Mbps_sh } \\ 3 & 1.90 & 3.44 & 5.62 & 8.44 \\ 10 & 2.95 & 3.30 & 6.70 & 7.20 \\ 25 & 2.98 & 3.01 & 7.03 & 6.93 \\ 50 & 3.06 & 2.85 & 6.81 & 6.84 \\ 75 & 3.08 & 2.80 & 6.87 & 6.96 \\ 100 & 2.99 & 2.78 & 6.85 & 6.88 \\ 150 & 2.98 & 2.70 & 6.80 & 6.81 \\ 200 & 2.96 & 2.70 & 6.82 & 6.97 \\ 300 & 2.94 & 2.70 & 6.83 & 6.86 \\ 400 & 2.86 & 2.62 & 6.83 & 6.84\end{array}$

A. 4 Conclusion simulations

From these simulations, we see that the shaped traffic has much higher throughput compared to the unshaped traffic when the CBS was small. When the CBS is large, the shaped traffic performs slightly less than the unshaped traffic due to the delay in the shaper. The G-trRAS solves this problem. Additional simulation results may be found in [Cnodder] 


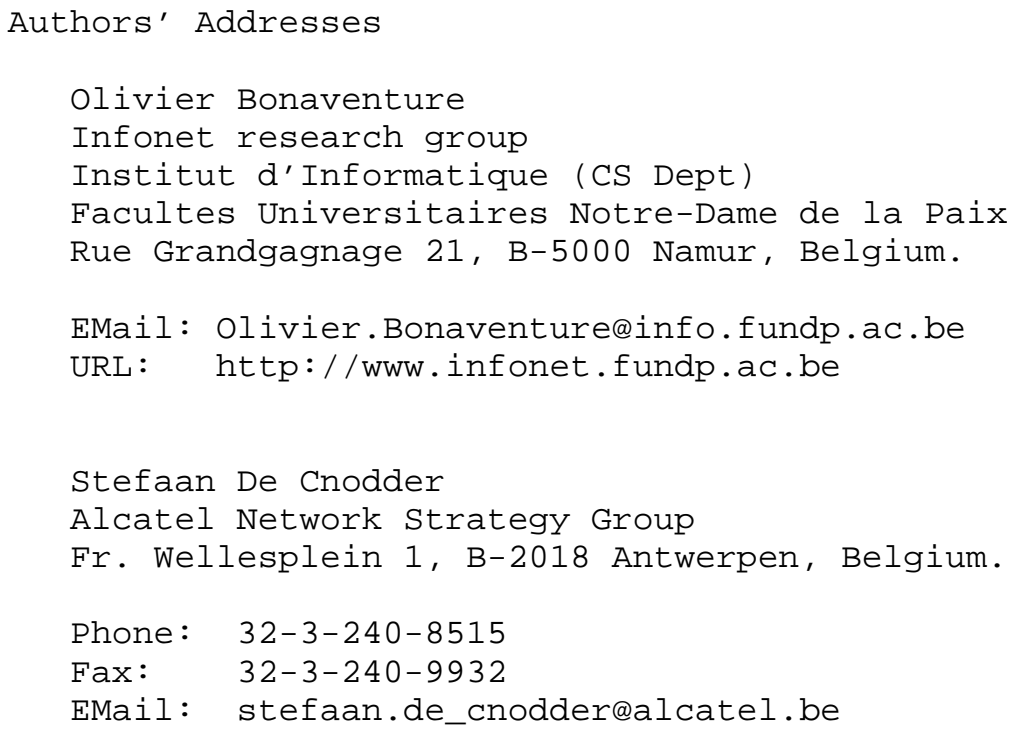


Full Copyright statement

Copyright (C) The Internet Society (2000). All Rights Reserved.

This document and translations of it may be copied and furnished to others, and derivative works that comment on or otherwise explain it or assist in its implementation may be prepared, copied, published and distributed, in whole or in part, without restriction of any kind, provided that the above copyright notice and this paragraph are included on all such copies and derivative works. However, this document itself may not be modified in any way, such as by removing the copyright notice or references to the Internet society or other Internet organizations, except as needed for the purpose of developing Internet standards in which case the procedures for copyrights defined in the Internet Standards process must be followed, or as required to translate it into languages other than English.

The limited permissions granted above are perpetual and will not be revoked by the Internet society or its successors or assigns.

This document and the information contained herein is provided on an "AS IS" basis and THE INTERNET SOCIETY AND THE INTERNET ENGINEERING TASK FORCE DISCLAIMS ALL WARRANTIES, EXPRESS OR IMPLIED, INCLUDING BUT NOT LIMITED TO ANY WARRANTY THAT THE USE OF THE INFORMATION HEREIN WILL NOT INFRINGE ANY RIGHTS OR ANY IMPLIED WARRANTIES OF MERCHANTABILITY OR FITNESS FOR A PARTICULAR PURPOSE.

Acknowledgement

Funding for the RFC Editor function is currently provided by the Internet Society. 